\title{
Gestión de proyectos de desarrollo de complementos alimenticios en cápsula de gelatina blanda.
}

\author{
Esther Martino Zamarriego ${ }^{1,2}$, Blanca Rodríguez² \\ 1. Unidad de Bioquímica y Biología Molecular, Departamento de Biología de Sistemas, Facultad de Medicina y Ciencias \\ de la Salud, Universidad de Alcalá, 28871 Alcalá de Henares, Madrid, España. 2. HC Clover, PS S.L. - Aplicaps, Calle \\ Alicante 8, 28500 Arganda del Rey, Madrid, España.
}

Palabras clave: HC Clover; gestión de proyectos; complementos alimenticios; cápsula de gelatina blanda; ficha técnica

\section{Resumen}

HC Clover es una empresa española de fabricación y desarrollo por contrato, que destaca por el desarrollo de complementos alimenticios en cápsula de gelatina blanda. Cada proyecto de desarrollo de complementos alimenticios es asignado a un gestor de proyectos, que lo dirige desde la fase inicial hasta la final. Para el desarrollo del producto, el cliente escoge la fórmula y el diseño asesorado por los departamentos de nuevas formulaciones y presupuestos. El proyecto presupuestado con el cliente se transfiere al gestor de proyectos, el cual da de alta en el servidor los códigos necesarios para la identificación del producto, garantizando su trazabilidad. A continuación, el gestor de proyectos informa de la fórmula y de las especificaciones acordadas con el cliente al departamento de desarrollo galénico, que se encarga de transformar los ingredientes activos farmacéuticos en la forma farmacéutica de cápsula de gelatina blanda. Tras finalizar el desarrollo galénico, se emite el Drug Master Formula, que recoge la fórmula y las especificaciones físicas del producto. A partir del DMF firmado, el gestor de proyectos elabora la ficha técnica del producto, la cual recoge todas sus especificaciones. Tras ser firmada por el cliente, las cápsulas de gelatina blanda se fabrican utilizando el proceso de rotary-die. Para garantizar la calidad del producto, una vez fabricado y acondicionado, el departamento de control de calidad realiza estudios de estabilidad para determinar su vida útil. Además, el equipo de Regulatory se encarga de gestionar el etiquetado y la notificación de puesta en el mercado del producto. En conclusión, la complejidad del proceso de desarrollo de complementos alimenticios, exige la presencia de un equipo de trabajo cualificado que trabaje bajo la normativa vigente, un buen sistema de calidad y la figura del gestor de proyectos que coordine y supervise todas las fases por las que pasa el proyecto.

Cita: Martino Zamarriego, Esther; Rodríguez, Blanca (2019) Gestión de proyectos de desarrollo de complementos alimenticios en cápsula de gelatina blanda. dianas 8 (2): e201909fa04. ISSN 1886-8746 (electronic) journal.dianas.e201909fa04 http://www3.uah.es/dianas?e201909fa04. URI http://hdl.handle.net/10017/15181

Copyright: $\odot$ Martino-Zamarriego E, Rodríguez B. Algunos derechos reservados. Este es un artículo openaccess distribuido bajo los términos de una licencia de Creative Commons Reconocimiento-NoComercialSinObraDerivada 4.0 Internacional. http://creativecommons.org/licenses/by-nc-nd/4.0/

\section{Introducción}

HC Clover es una empresa privada española de fabricación y desarrollo por contrato (CDMO) [1]. Actúan como socios para la industria de la salud, farmacéutica y veterinaria, al ser uno de los principales fabricantes del sur de Europa de la forma farmacéutica de cápsula de gelatina blanda [1]. No comercializan productos, solo ofrecen servicios, ya que los productos pertenecen a sus clientes [1]. HC Clover presenta dos líneas de negocio: Aplicaps by Clover, especializada en cápsulas de gelatina blanda; y Laboratorios Gramar, especializada en otras formas farmacéuticas y de acondicionamiento primario y secundario [1].

Las cápsulas de gelatina blanda son una forma farmacéutica formada por una cubierta de gelatina blanda que contiene los ingredientes activos en solución, suspensión o pasta [2]. Este tipo de cápsulas están formadas por una combinación de gelatina, agua, plastificante (glicerina y/o sorbitol) e ingredientes opcionales como colorantes, opacificantes, saborizantes, edulcorantes y conservantes [2,3]. Presentan grandes ventajas frente a otras formas farmacéuticas: mejoran la biodisponibilidad de los compuestos poco solubles en agua y/o permeables al tracto gastrointestinal (clases II, III y IV del Sistema de Clasificación Biofarmacéutica (BCS)), gracias a que el ingrediente activo farmacéutico (API) se encuentra solubilizado; al estar selladas herméticamente, mejoran la uniformidad de contenido en compuestos que se administran a dosis muy bajas, mejorando así la precisión en la dosificación; son farmacológicamente más activas, ya que la ruptura de la cápsula se produce a los 3-5 minutos, lo cual da lugar a una rápida absorción; protegen al API de la oxidación por radiación ultravioleta (UV), etc. [1] Estas ventajas han conseguido que la industria farmacéutica cada vez se decante más por este tipo de formulación [1]. 
HC Clover destaca por el desarrollo de complementos alimenticios en cápsula de gelatina blanda [1]. Ofrece un servicio integral para cada producto, que va desde la idea del producto, hasta obtener un producto listo para su comercialización [1] (Figura 1).

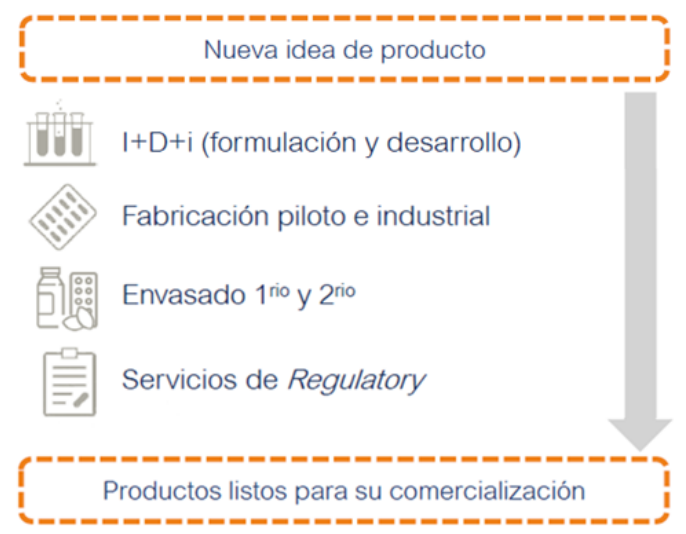

Figura 1.- Servicio que ofrece HC Clover para el desarrollo de cada producto.

Cada proyecto es asignado a un gestor de proyectos, el cual lo dirige desde la fase inicial hasta la fase final. El gestor de proyectos actúa como intermediario entre el cliente y la empresa, elabora y mantiene actualizada la documentación técnica del producto, y permanece al tanto de cada una de las fases por las que va pasando el proyecto, midiendo su evolución y responsabilizándose del cumplimiento de los plazos y presupuestos acordados.

El objetivo de esta memoria es describir el hilo de trabajo que sigue HC Clover para el desarrollo de complementos alimenticios en cápsula de gelatina blanda, destacando las funciones del gestor de proyectos en el proceso.

\section{I+D+i (formulación y desarrollo)}

Según la Directiva 2002/46/CE del Parlamento Europeo, transpuesta a nuestro ordenamiento jurídico por el Real Decreto 1487/2009, de 26 de septiembre, relativo a los complementos alimenticios, los complementos alimenticios se definen como productos alimenticios cuyo fin es complementar la dieta normal, consistentes en fuentes concentradas de nutrientes (vitaminas y minerales), $\mathrm{u}$ otras sustancias con un efecto nutricional o fisiológico (aminoácidos, ácidos grasos esenciales, fibras y diversas plantas y extractos de hierbas), en forma simple o combinada, y comercializados en forma dosificada [4]. Los complementos alimenticios no deben consumirse como sustitutos de una dieta equilibrada, ya que, en condiciones normales, una dieta adecuada proporciona todos los nutrientes necesarios para el normal desarrollo y mantenimiento de un organismo sano [4]. Sin embargo, en determinadas ocasiones, se recomienda el consumo de complementos alimenticios, ya que, en la práctica, esta situación ideal no se cumple para todos los nutrientes, ni para todos los grupos de población [4].

Al ser considerados productos alimenticios, los complementos alimenticios deben cumplir todos los aspectos pertinentes de la legislación alimentaria en cuanto a su composición, fabricación, distribución y control [5]. Su regulación, por tanto, difiere bastante, en cuanto a fabricación, puesta en el mercado y promoción, de la de los medicamentos [6].

\section{Formulación}

El desarrollo de un complemento alimenticio comienza con una idea que puede partir del cliente, de la compañía o del trabajo conjunto entre la compañía y el cliente. HC Clover trabaja con tres tipos de fórmulas diferentes, de modo que el cliente escoge la que mejor se adapte a él: fórmulas stock (commodities), productos desarrollados por HC Clover a precios competitivos y con cortos plazos de entrega; fórmulas exclusivas, productos desarrollados por $\mathrm{HC}$ Clover con ingredientes premium patentados, exclusivos para los clientes, que poseen ensayos clínicos; y fórmulas personalizadas, productos con fórmulas adaptadas a las necesidades del cliente [1].

Para la elección de la fórmula y el diseño de los productos, el departamento de nuevas formulaciones, junto con el de presupuestos, ofrece un asesoramiento profesional para ayudar a alcanzar los objetivos de negocio del cliente. Si el cliente quiere desarrollar una fórmula personalizada, en función del área de salud a la que quiera dirigir el complemento alimenticio, escoge los APIs basándose en el Reglamento (UE) $\mathrm{N}^{\circ} 432 / 2012$ de la Comisión, de 16 de mayo de 2012, por el que se establece una lista de declaraciones autorizadas de propiedades saludables de los alimentos distintas de las relativas a la reducción del riesgo de enfermedad y al desarrollo y la salud de los niños [7]. 
Solo podrán utilizarse en la fabricación de complementos alimenticios las vitaminas y minerales recogidas en el anexo I, y en las formas enumeradas en el anexo II, del Reglamento (CE) N N $^{\circ} 1170 / 2009$ de la Comisión, de 30 de noviembre de 2009, por el que se modifican la Directiva 2002/46/CE del Parlamento Europeo y del Consejo y el Reglamento (CE) No 1925/2006 del Parlamento Europeo y del Consejo en lo relativo a las listas de vitaminas y minerales y sus formas que pueden añadirse a los alimentos, incluidos los complementos alimenticios; y las sustancias que hayan sido aprobadas por la Autoridad Europea de Seguridad Alimentaria (EFSA) sobre la base de los criterios mencionados para su utilización en la fabricación de alimentos destinados a lactantes y a niños de corta edad, y otros alimentos para usos nutricionales particulares [8, 9]. Para garantizar que los complementos alimenticios complementen la ingesta de vitaminas y minerales en la dieta habitual, estas sustancias deben encontrarse en cantidades significativas [4]. Sin embargo, considerando que una ingesta excesiva y continuada de complementos alimenticios puede tener efectos perjudiciales para la salud, se han establecido niveles máximos para ciertos nutrientes de forma que se garantice que la utilización normal de estos productos, de acuerdo con las instrucciones de uso dadas por el fabricante, no presente peligro para los consumidores [4].

Para comercializar en España complementos alimenticios que contengan ingredientes distintos de las vitaminas y minerales recogidos en las listas autorizadas, se aplica el principio de reconocimiento mutuo [10]. Este principio permite la comercialización de estos productos en España, únicamente si se acredita que el producto se comercializa legalmente en otro Estado miembro de la Unión Europea [10]. Por tanto, no es posible aplicar este principio a productos provenientes de países no pertenecientes a la Unión Europea [10].

\section{Códigos}

Una vez establecidos los APIs y el acondicionamiento del producto, al gestor de proyectos se le transfiere el proyecto presupuestado con el cliente. Para garantizar la trazabilidad del producto, el gestor de proyectos da de alta en el servidor los códigos necesarios para su identificación: código de las materias primas nuevas (en caso de que las haya), código del granel, código de los materiales de acondicionamiento y código del producto terminado. Como los complementos alimenticios en cápsula de gelatina blanda se fabrican en Aplicaps y se acondicionan en Laboratorios Gramar, se crean códigos en el servidor de Aplicaps, Fénix, y en el servidor de Laboratorios Gramar, SAP. Los códigos son diferentes y comienzan con números identificativos diferentes.

\section{Desarrollo galénico}

A continuación, el gestor de proyectos informa de la fórmula y de las especificaciones acordadas con el cliente al departamento de desarrollo galénico. Desarrollo galénico se encarga de transformar los APIs escogidos por el cliente en la forma farmacéutica de cápsula de gelatina blanda, permitiendo un máximo aprovechamiento terapéutico: solubilidad, biodisponibilidad y permeabilidad óptimas. Así, se establece la fórmula del producto, donde los APIs van acompañados de: los excipientes y la envolvente.

Tras finalizar el desarrollo galénico, se emite el Drug Master Formula (DMF). Este documento recoge el nombre del producto, la caducidad, la fórmula (cualitativa y cuantitativa) y las especificaciones físicas de la cápsula (formato, color, peso medio, uniformidad de masa del contenido y tiempo de desintegración). Sin embargo, los datos recogidos en el DMF pueden sufrir algunas modificaciones. La fórmula no quedará $100 \%$ definida hasta la fabricación del primer lote piloto o la fabricación del lote industrial, pudiendo haber una variación de un $\pm 10 \%$ de los datos de excipientes y envolvente.

El gestor de proyectos envía el DMF al cliente para que lo firme, si está conforme. Una vez el DMF está firmado, el gestor de proyectos actualiza el servidor con la fórmula final del producto, y se podría comenzar a fabricar el granel.

\section{Ficha técnica}

A partir del DMF firmado, el gestor de proyectos puede comenzar a elaborar la ficha técnica del producto. Este es un documento que recoge todas las especificaciones del producto: nombre, loteado, caducidad, fórmula, especificaciones físicas, valores nutricionales, análisis microbiológicos, acondicionamiento, análisis de metales pesados, alérgenos, ausencia de organismos modificados genéticamente (OMG), ausencia de encefalopatía espongiforme bovina y otras encefalopatías espongiformes transmisibles (BSE/TSE), y conservación.

Para la elaboración de la ficha técnica, el gestor de proyectos tiene que revisar la documentación de las materias primas que componen el producto, para asegurarse de que cumplen con las especificaciones declaradas en la ficha técnica. La documentación de materias primas se archiva en el servidor de la empresa. En caso de emplearse una materia prima nueva, el gestor de proyectos solicita la documentación que necesita para elaborar la ficha técnica al departamento de control de calidad, que se encarga de pedir la documentación al proveedor de la materia prima. 


\section{Loteado}

Según el Real Decreto 1808/1991, de 13 de diciembre, por el que se regulan las menciones o marcas que permiten identificar el lote al que pertenece un producto alimenticio, se entiende por lote a un conjunto de unidades de venta de un producto alimenticio, producido, fabricado o envasado en circunstancias prácticamente idénticas [11]. La indicación del lote de un producto alimenticio es necesaria para proporcionar información sobre su identidad, la cual es especialmente útil cuando surgen disputas o cuando los productos alimenticios presentan un peligro para la salud de los consumidores [11]. Por este motivo, salvo excepciones, solo se podrá comercializar un producto alimenticio si va acompañado de la indicación del lote [11].

HC Clover indica en la ficha técnica del producto la manera en la que lotea: X0000 (letra correspondiente al año de fabricación + lotes consecutivos fabricados). La indicación del lote en un producto alimenticio tiene que ir precedida de la letra "L", salvo en los casos en los que se distinga claramente de las demás indicaciones del etiquetado [11]. Cuando los productos estén envasados, la indicación del lote figurará en el envase o en una etiqueta unida a éste; y cuando no estén envasados, figurará en el embalaje, en el recipiente o en los documentos comerciales pertinentes [11]. En todos los casos, debe ser fácilmente visible, claramente legible e indeleble [11].

\section{Información nutricional}

El etiquetado sobre la información nutricional es obligatorio para la mayoría de los alimentos transformados, ya que informa a los consumidores sobre la composición de los alimentos [12]. Las normas relativas a la información nutricional del Reglamento (UE) No 1169/2011, del Parlamento Europeo y del Consejo, de 25 de octubre de 2011, sobre la información alimentaria facilitada al consumidor, no se aplican a los complementos alimenticios, ya que estos tienen su normativa específica [12].

En los complementos alimenticios, se debe declarar en la etiqueta la cantidad de nutrientes o sustancias con un efecto nutricional o fisiológico contenida en el producto, de forma numérica y por dosis de producto diaria recomendada por el fabricante [4]. Para las vitaminas y minerales se utilizarán las unidades indicadas en el anexo I del Reglamento (CE) No 1170/2009; y se expresarán en porcentaje de los valores de referencia mencionados en el anexo XIII del Reglamento (UE) No 1169/2011 [4, 8, 9, 12]. La información que se quiera aportar de forma voluntaria sobre el producto respecto a nutrientes $\mathrm{u}$ otras sustancias contenidas en él, se debe ajustar al Reglamento (UE) $\mathrm{N}^{\circ} 1169 / 2011$ y al Reglamento (CE) $\mathrm{N}^{\circ}$ 1924/2006 del Parlamento Europeo y del Consejo, de 20 de diciembre de 2006, relativo a las declaraciones nutricionales y de propiedades saludables en los alimentos [12-14].

De este modo, HC Clover además indica el aporte nutricional de sus productos. El aporte nutricional es calculado por el gestor de proyectos a partir de la información nutricional suministrada por el proveedor de cada una de las materias primas que lo componen.

\section{Análisis microbiológicos}

Los riesgos microbiológicos de los productos alimenticios constituyen una de las principales fuentes de enfermedades de origen alimentario para las personas [15]. El Reglamento (CE) $N^{\circ} 2073 / 2005$ de la Comisión, de 15 de noviembre de 2005, relativo a los criterios microbiológicos aplicables a los productos alimenticios, establece que los productos alimenticios no deben contener microorganismos, ni sus toxinas o metabolitos, en cantidades que supongan un riesgo inaceptable para la salud humana [15]. La seguridad de los productos alimenticios se garantiza principalmente mediante un enfoque preventivo, como la adopción de buenas prácticas de higiene y la aplicación de procedimientos basados en los principios Análisis de Peligros y Puntos Críticos de Control (APPCC) [15]. En consecuencia, es conveniente fijar criterios microbiológicos que definan la aceptabilidad de los procesos, así como criterios microbiológicos para la seguridad de los alimentos que establezcan un límite por encima del cual un producto alimenticio deba considerarse contaminado [15]. En HC Clover todos sus productos cumplen con el contenido máximo de microorganismos establecido por la Farmacopea Europea (Ph. Eur.) (Tabla 1).

\begin{tabular}{|c|c|}
\hline MICROORGANISMOS & CONTENIDO MÁXIMO \\
\hline Aerobios mesófilos a $\mathbf{3 0}^{\circ} \mathbf{C}$ & $10^{4} \mathrm{ufc} / \mathrm{g}$ \\
\hline Mohos y levaduras & $10^{2} \mathrm{ufc} / \mathrm{g}$ \\
\hline Enterobacterias & $10^{2} \mathrm{ufc} / \mathrm{g}$ \\
\hline Escherichia coli & Ausencia $1 \mathrm{~g}$ \\
\hline Salmonella & Ausencia $10 \mathrm{~g}$ \\
\hline Staphylococcus aureus & Ausencia $1 \mathrm{~g}$ \\
\hline Listeria monocytogenes & Ausencia $25 \mathrm{~g}$ \\
\hline
\end{tabular}

Tabla 1.- Contenido máximo de microorganismos en complementos alimenticios según Ph. Eur. 


\section{Acondicionamiento}

En HC Clover las cápsulas de gelatina blanda pueden presentarse a granel o como producto terminado, teniendo en cuenta que el acondicionamiento tiene que ser compatible con la forma galénica. Cuando las cápsulas se presentan a granel, se almacenan en bolsas de plástico transparentes cerradas con una brida de plástico. El número de cápsulas por bolsa varía en función del formato y tamaño de la cápsula. Por otro lado, cuando las cápsulas van acondicionadas, presentan un acondicionamiento primario (en botes de plástico o vidrio, o en blíster) con o sin acondicionamiento secundario (estuche con o sin folleto).

\section{Metales pesados}

Los metales pesados son un grupo de elementos químicos, emitidos principalmente por la actividad industrial y minera, que presentan una densidad relativamente alta y cierta toxicidad para el ser humano, al no ser química ni biológicamente degradables [16]. Los metales pesados tóxicos más conocidos son el mercurio, el plomo y el cadmio, de modo que los niveles máximos de estos metales pesados deben ser seguros y tan bajos como sea razonablemente posible, conforme a unas buenas prácticas de fabricación $[16,17]$. Por este motivo, el Reglamento (CE) No 629/2008 de la Comisión, de 2 de julio de 2008, que modifica el Reglamento (CE) No $1881 / 2006$ por el que se fija el contenido máximo de determinados contaminantes en los productos alimenticios, establece los contenidos máximos para el plomo, el cadmio y el mercurio en complementos alimenticios [17] (Tabla 2). HC Clover no realiza controles de metales pesados por lote fabricado, estos controles son exigidos a los proveedores de materias primas.

\begin{tabular}{|c|c|}
\hline METALES PESADOS & CONTENIDO MÁXIMO (mg/kg) \\
\hline Plomo & 3 \\
\hline Cadmio & 1 \\
\hline Mercurio & 0,1 \\
\hline
\end{tabular}

Tabla 2.- Contenido máximo de metales pesados (plomo, cadmio, mercurio) en complementos alimenticios. [17]

\section{Alérgenos}

Siempre debe facilitarse al consumidor la información sobre la presencia de alérgenos en los alimentos [12]. En el anexo II del Reglamento (UE) No 1169/2011, se encuentra la lista de sustancias o productos alimenticios que causan alergias o intolerancias, la cual ha sido elaborada sobre la base de dictámenes científicos adoptados por la EFSA [12]. En los alimentos envasados, la información sobre los alérgenos deberá aparecer en la lista de ingredientes, debiendo destacarse mediante una composición tipográfica (tipo de letra, estilo o color de fondo) que la diferencie claramente del resto de la lista de ingredientes [12]. En ausencia de lista de ingredientes, debe incluirse la mención "contiene" seguida de la sustancia o producto que figura en el anexo II del Reglamento (UE) $N^{0}$ 1169/2011 [12]. Sin embargo, no serán necesarias estas menciones en aquellos casos en los que la denominación del alimento haga referencia claramente a la sustancia o producto de que se trate [12]. En el caso de los alimentos sin envasar, se está autorizado informar de la presencia de alérgenos mediante cualquier medio de comunicación [12].

HC Clover no realiza controles de alérgenos por lote fabricado, estos controles son exigidos a los proveedores de materias primas. Sin embargo, en la línea de fabricación de HC Clover puede haber trazas de otros productos, pudiéndose producir fenómenos de contaminación cruzada. Por tanto, si el cliente quiere declarar su producto como libre de alérgenos, tiene que hacer un análisis por cada lote de producto final.

\section{Organismos modificados genéticamente}

Según la Directiva 2001/18/CE, de 12 de marzo de 2001, sobre la liberación intencional en el medio ambiente de OMG y por la que se deroga la Directiva 90/220/CEE del Consejo, los OMG son organismos, con excepción de los seres humanos, cuyo material genético ha sido modificado de una manera que no se produce naturalmente en el apareamiento ni en la recombinación natural [18]. Por tanto, según el Reglamento (CE) No 1829/2003 del Parlamento Europeo y del Consejo, de 22 de septiembre de 2003, sobre alimentos y piensos modificados genéticamente, los alimentos modificados genéticamente son aquellos que contienen o están compuestos por OMG o han sido producidos a partir de ellos [19]. Todos los alimentos cuya comercialización se inicia en la Unión Europea, incluidos los alimentos modificados genéticamente, son sometidos a evaluaciones de seguridad alimentaria que garantizan que su consumo es seguro [19]. Sin embargo, la legislación europea obliga a que se etiqueten todos aquellos alimentos cuyo contenido en OMG supere el 0,9\%, para facilitar la libre elección del consumidor [19]. HC Clover no trabaja con OMG ni con materias primas producidas a partir de ellos. Esto le permite declarar que sus productos son libres de OMG. 


\section{Encefalopatías espongiformes transmisibles}

Las encefalopatías espongiformes transmisibles (EETs) son una serie de enfermedades nerviosas, del hombre y de los animales, de carácter degenerativo y de evolución mortal [20]. La hipótesis más aceptada en cuanto a su etiología defiende que las EETs están causadas por priones, siendo estos la forma alterada de una proteína intraneuronal y de otros tejidos (células del sistema inmunológico), que ha perdido su función normal pero que ha adquirido la capacidad de transformar la forma normal en patológica [20]. Dentro de las EETs se encuentra la encefalopatía espongiforme bovina (EEB), que ocurre en el ganado bovino [20]. La transmisión de la EEB al humano, evoluciona en la enfermedad de Creutzfeldt-Jakob (vECJ), lo cual puede ocurrir como consecuencia del consumo de productos cárnicos contaminados [21].

Para prevenir la infección en los animales y el humano, muchos países han reforzado las medidas de control, como la eliminación de los tejidos que puedan contener una cantidad significativa de infectividad de EEB (cerebro, ojos, médula espinal, cráneo, columna vertebral, tonsilas e íleon distal) y la prohibición del uso de proteínas animales procesadas en los piensos [22]. HC Clover trabaja con materias primas con certificado de ausencia de BSE/TSE. Aunque en la actualidad, como resultado de la implementación exitosa de medidas de control eficaces, la prevalencia, el impacto sanitario mundial y el riesgo para la salud pública de la EEB clásica es extremadamente baja [21].

\section{Conservación}

Las condiciones de conservación y/o de utilización deben ser indicadas cuando los alimentos requieran unas condiciones especiales [12]. Una vez abierto el envase, cuando proceda, también deben indicarse las condiciones y/o la fecha límite de consumo [12]. Salvo excepciones, HC Clover establece que sus productos deben conservarse en un lugar fresco y seco, entre temperaturas de $15-25^{\circ} \mathrm{C}$ y $\mathrm{HR}<60 \%$.

Además de la documentación de materias primas necesaria para la elaboración de la ficha técnica del producto, el cliente a veces solicita que se le envíe documentación adicional de la materia prima, como puede ser: origen, diagrama de flujo, declaración de no irradiación, declaración de ausencia de nanomateriales, etc.

Una vez finalizada la ficha técnica del producto, se envía al cliente para que la apruebe. Cuando el cliente firma la ficha técnica, se pueden empezar a gestionar los materiales de acondicionamiento. Aunque se puede comenzar a fabricar el granel cuando el DMF está aprobado, generalmente se espera a que la ficha técnica esté firmada. Finalmente, cuando el cliente firma el diseño de los materiales de acondicionamiento, también se pueden comenzar a fabricar.

\section{Fabricación piloto e industrial}

Para la fabricación de un nuevo producto, primero se hace una fabricación en una planta piloto, es decir, una planta de proceso a escala reducida. El fin es obtener información sobre el proceso para determinar si es técnica y económicamente viable, así como establecer los parámetros de operación óptimos para la posterior fabricación a escala industrial.

En HC Clover, las cápsulas de gelatina blanda se forman, rellenan y sellan utilizando el proceso de rotary-die [1]. En primer lugar se compran las materias primas, las cuales se mantienen en cuarentena durante un tiempo. Una vez son aprobadas, pasan por la cabina de pesadas, donde se pesan para la posterior fabricación de la envolvente y el contenido. Para la fabricación de la envolvente, se calienta y disuelve la gelatina en agua, lo cual va seguido de la adición del plastificante [22]. Una vez la gelatina está completamente disuelta, se pueden agregar otros componentes como colorantes, opacificantes, saborizantes, edulcorantes y conservantes [2,22]. La masa uniforme de gelatina caliente se suministra a la máquina de encapsulación pasando por un tambor de enfriamiento, de modo que se forman dos cintas de gelatina separadas, donde cada una proporciona la mitad de la cápsula de gelatina blanda [22]. Por otro lado, la fabricación del contenido implica la disolución de los ingredientes en un vehículo líquido no acuoso, dando lugar a un producto final homogéneo [22].

La máquina de encapsulación tiene dos cilindros que giran en dirección contraria [22]. Cada uno de los cilindros recibe continuamente una cinta de gelatina que se corta en una de las dos mitades de la cápsula de gelatina blanda [22]. Estas mitades salen juntas durante la rotación, y justo antes de que los cilindros opuestos se unan, un inyector llena con el contenido cada una de las mitades de la cápsula [22]. A medida que giran los cilindros, las dos mitades se sellan dando lugar a la cápsula [22] (Figura 2).

Después de la encapsulación, las cápsulas se secan en un túnel de secado con una temperatura elevada y un gran volumen de aire forzado [3]. A continuación, se transfieren a bandejas y se colocan en una sala de secado de baja humedad [3]. Una vez se han secado, pasan a la sala de inspección, y aquellas cápsulas que se han fabricado correctamente ya pueden ser acondicionadas. Tras pasar una cuarentena, el producto se libera y se ubica en el almacén hasta su posterior expedición. 


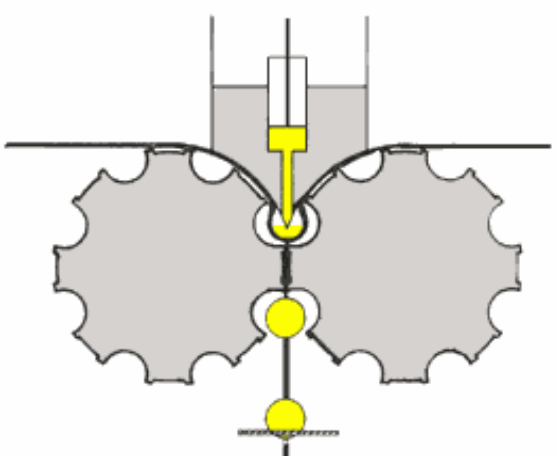

Figura 2.- Proceso de rotary-die para la fabricación de cápsulas de gelatina blanda.

Uno de los requisitos de calidad es que el producto sea suficientemente estable (química y físicamente) para poder cumplir con la información indicada en la etiqueta durante toda su vida útil, siempre que se almacene y utilice de acuerdo con las indicaciones de la etiqueta [23]. Para alcanzar el objetivo de calidad, es necesaria la participación y compromiso de todos los participantes en las etapas de fabricación, almacenamiento y distribución, y un buen sistema integrado de Buenas Prácticas de Fabricación y Distribución, Garantía de Calidad y Control de Calidad [5]. Por este motivo, una vez el producto ha sido fabricado y acondicionado interviene el departamento de Control de Calidad.

Control de calidad se encarga de asegurar que los productos cumplen con los requisitos mínimos de calidad, evitando la presencia de errores. Uno de los controles que realiza HC Clover son los estudios de estabilidad basados en las normas establecidas por el Consejo Internacional de armonización de los requisitos técnicos para el registro de medicamentos de uso humano (normas $\mathrm{ICH}$ ). En el presupuesto inicial el cliente determina si quiere hacer un estudio de estabilidad a su producto y los detalles del mismo.

\section{Estudio de estabilidad}

Un estudio de estabilidad es una serie de pruebas que se realizan para determinar la vida útil de un producto, es decir, el periodo de tiempo durante el cual un producto terminado conserva sus propiedades en determinadas condiciones de envase y almacenamiento [23]. Estos estudios aseguran que no se producen variaciones organolépticas, físicas, químicas o microbiológicas durante la vida útil propuesta [23]. Las pruebas de estabilidad se realizan en el recipiente y envase definitivos, y en las condiciones climáticas predominantes de la zona en la que se usará el producto [24].

A la hora de realizar un estudio de estabilidad, el gestor de proyectos se encarga de redactar un protocolo que recoja todas las especificaciones sobre el estudio: objetivo y alcance del estudio, composición cuantitativa del producto, condiciones de conservación del producto, distribución del producto en cámaras, y determinaciones y condiciones. Este protocolo se envía al cliente, y una vez que lo firme se podrá comenzar el estudio de estabilidad.

Para los productos que contienen principios activos más o menos estables, se toman muestras de dos lotes de producción diferentes; por el contrario, si los productos contienen principios activos que se degradan con facilidad o sustancias con escasos datos de estabilidad, se toman muestras de tres lotes [24]. Los lotes de los que se obtienen las muestras tienen que ser representativos del proceso de fabricación, y siempre que sea posible, deberán estar fabricados con lotes diferentes de principios activos [24].

Los estudios de estabilidad se pueden realizar acelerados o a tiempo real [23]. Los estudios acelerados implican un almacenamiento del producto a temperaturas considerablemente superiores a las del almacenamiento esperado en el mercado [23]. Estos estudios se basan en la presunción de que la velocidad de una reacción química se duplica con aproximadamente cada incremento de $10^{\circ} \mathrm{C}$ de temperatura, de modo que proporcionan información sobre la vida útil en un plazo de tiempo relativamente corto [23]. Sin embargo, estos estudios ofrecen una precisión limitada a la hora de predecir la vida útil, por ello se recomienda que también se realicen estudios de estabilidad a tiempo real para confirmar los resultados del estudio de estabilidad acelerado [23]. Los estudios a tiempo real se realizan con productos almacenados en las mismas condiciones medioambientales que las partidas comerciales, $\mathrm{y}$ durante un periodo de tiempo similar a la vida útil de dichas partidas [23].

Durante los estudios de estabilidad, se van recogiendo datos en distintos puntos del tiempo [23]. HC Clover solo realiza los análisis organolépticos y físicos de los productos, los análisis químicos y microbiológicos los realiza otra empresa. Los resultados obtenidos a cada tiempo se envían al gestor de proyectos para que realice el correspondiente informe de estabilidad, donde se detalla el plan de estudio, así como los resultados y conclusiones obtenidas. El informe de estabilidad que se realiza a cada tiempo se envía al cliente para que lo firme si está conforme con los resultados obtenidos en el estudio. 
Si se produce una variación significativa en el producto de modo que no logre satisfacer las especificaciones establecidas, por lo general, no será necesario continuar con el estudio [23]. En caso de que el estudio de estabilidad se esté realizando de forma simultánea a la distribución del producto en comercios, el fabricante del producto debe evaluar la situación para determinar las acciones a adoptar: cancelación de la distribución, nuevo etiquetado con una vida útil más corta, notificar a los clientes, retirada del material previamente distribuido o la retirada completa del producto [23]. Por otro lado, si no se observan variaciones significativas durante el estudio, es posible que se pueda prolongar la vida útil del producto más allá de la inicialmente esperada [23].

\section{Servicios de Regulatory}

Para garantizar la total legalidad del producto, el equipo de Regulatory ayuda a superar las barreras administrativas y regulatorias, trabajando sobre la legislación vigente a la que se adscribe el producto [1]. Entre sus funciones destaca la realización del etiquetado del producto y la notificación de puesta en el mercado.

\section{Etiquetado}

El etiquetado de los complementos alimenticios debe de incluir la siguiente información alimentaria obligatoria general: la denominación de complemento alimenticio; la lista de ingredientes; las sustancias o productos que pueden causar alergias o intolerancias; la cantidad de determinados ingredientes o determinadas categorías de ingredientes; la cantidad neta del alimento; la fecha de duración mínima o fecha de caducidad; las condiciones especiales de conservación y/o las condiciones de utilización; el nombre o la razón social y la dirección del operador de la empresa alimentaria; el país de origen o lugar de procedencia cuando así esté previsto en el artículo 26 del Reglamento (UE) No 1169/2011; el modo de empleo en caso de que, en ausencia de esta información, fuera difícil hacer un uso adecuado del alimento; y la información nutricional [12].

En cuanto al etiquetado específico de los complementos alimenticios, en la etiqueta deben figurar además obligatoriamente los siguientes datos: la denominación de las categorías de nutrientes o sustancias que caractericen el producto, o una indicación relativa a la naturaleza de dichos nutrientes o sustancias; la dosis del producto recomendada para consumo diario; la advertencia de no superar la dosis diaria expresamente recomendada; la afirmación expresa de que los complementos alimenticios no deben utilizarse como sustitutos de una dieta equilibrada; la indicación de que el producto se debe mantener fuera del alcance de los niños más pequeños; y las advertencias de seguridad establecidas en la tercera columna del anexo del Real Decreto 130/2018, de 16 de marzo, por el que se modifica el Real Decreto 1487/2009, de 26 de septiembre, relativo a los complementos alimenticios [4, 8]. Además, el etiquetado, la presentación y la publicidad de los complementos alimenticios no incluirán ninguna afirmación que declare o sugiera que una dieta equilibrada y variada no aporta las cantidades adecuadas de nutrientes, y no atribuirá a los complementos alimenticios la propiedad de prevenir, tratar o curar una enfermedad humana [4].

La información alimentaria obligatoria tiene que indicarse en un lugar destacado, de manera que sea fácilmente visible, claramente legible e indeleble [12]. Por ello, el tamaño mínimo de fuente es de 1,2 $\mathrm{mm}$; sin embargo, si la superficie máxima del envase es inferior a $80 \mathrm{~cm}^{2}$, el tamaño mínimo se reduce a $0,9 \mathrm{~mm}$ [12]. En el caso de los alimentos envasados, la información alimentaria obligatoria debe figurar directamente en el envase o en una etiqueta sujeta al mismo, o mediante otro medio siempre que se asegure el mismo nivel de información [12]. En caso de que el envase sea menor de $25 \mathrm{~cm}^{2}, 1 \mathrm{la}$ información nutricional no será obligatoria; y en caso de que en el envase la superficie más grande sea inferior a $10 \mathrm{~cm}^{2}$, no será necesario incorporar ni la información nutricional ni la lista de ingredientes [12]. Una o varias de estas menciones se podrán expresar mediante pictogramas y símbolos, en lugar de palabras y números, siempre que se asegure el mismo nivel de información [12]. Por otro lado, en los alimentos no envasados, solo será obligatoria la mención, a través de cualquier medio de comunicación, de las sustancias o productos que pueden causar alergias o intolerancias [12].

\section{Notificación de puesta en el mercado}

Para la comercialización de complementos alimenticios, las empresas alimentarias únicamente deben estar inscritas en la clave 26, actividad 25, del Registro General Sanitario de Empresas Alimentarias y Alimentos (RGSEAA) y notificar su puesta en el mercado con carácter previo o simultáneo a la primera puesta en el mercado [4, 25].

El responsable de la comercialización en España del producto (el fabricante, el responsable de la primera puesta en el mercado o el importador), debe notificar su puesta en el mercado nacional a las autoridades competentes [13]. Dicha notificación permite iniciar la comercialización del producto en España desde el día que se presenta [13]. Consiste en la presentación de un formulario con los datos necesarios sobre el 
producto y la empresa responsable del mismo, así como un ejemplar del etiquetado del complemento a comercializar [25].

La notificación de la puesta en el mercado nacional se presenta ante los órganos de la comunidad autónoma competente, en función del domicilio social del fabricante o el responsable de la primera puesta en el mercado [4]. Cuando el domicilio social del fabricante o del responsable de la primera puesta en el mercado se encuentra en la Comunidad de Madrid, como es el caso de HC Clover, la notificación se debe presentar ante la Consejería de Sanidad de la Comunidad de Madrid [13]. A continuación, la comunidad autónoma comunica a la Agencia Española de Consumo, Seguridad Alimentaria y Nutrición (AECOSAN) de la notificación [4]. Cuando el responsable no tiene establecido su domicilio social en España o cuando procede de terceros países, las notificaciones de los complementos alimenticios se presentan directamente ante el Ministerio de Sanidad, Servicios Sociales e Igualdad - AECOSAN [4, 13].

\section{Producto listo para su comercialización}

Cuando el producto está listo para su comercialización, el departamento de logística se encarga de gestionar el envío a la dirección acordada con el cliente. Para un mayor desempeño de la tarea de venta, HC Clover ofrece formación científica sobre el producto para los departamentos de Comercial y Marketing [1]. Además, los comerciales ayudan a establecer una estrategia de venta y promoción del producto dentro del sector [1].

Sin embargo, una vez en el mercado, la Administración podrá ponerse en contacto con el operador en caso de detectar alguna irregularidad del producto o su etiquetado, solicitándole la corrección o determinando la imposibilidad de continuar con la comercialización del producto [26]. La inexistencia de un plazo entre la comunicación de puesta en el mercado y la comercialización implica que, a diferencia de los medicamentos, en los complementos alimenticios las autoridades sanitarias no realizan ninguna evaluación de eficacia, seguridad, calidad y etiquetado antes de que el producto sea puesto a disposición de los consumidores [6]. Por tanto, la calidad del producto recae exclusivamente sobre el fabricante [6].

\section{Conclusión}

La complejidad del proceso de desarrollo de complementos alimenticios exige la presencia de un equipo de trabajo cualificado que trabaje bajo la normativa vigente, un buen sistema de calidad y la figura del gestor de proyectos que coordine y supervise todas las fases por las que pasa el proyecto, garantizando la protección de la salud de los consumidores y su necesidad de información.

\section{Bibliografía}

1. HC Clover Productos y Servicios, S.L. En Aplicaps. https://aplicaps.com/hc-clover/

2. Prasad Gullapalli, R. 2010. Soft gelatin capsules (softgels). Journal of pharmaceutical sciences. 99(10):4107:4148.

3. Raj, A. 2015. Soft gelatin capsules (softgels). PharmaTutor. 3(10):16-18.

4. Real Decreto $1487 / 2009$, de 26 de septiembre, relativo a los complementos alimenticios. Diario Oficial de la Unión Europea, núm. 244, de 9 de octubre de 2009, pág. 85370-85378.

5. Asociación de las Empresas de Dietéticos y Complementos Alimenticios. 2019. Guía de buenas prácticas de fabricación y distribución de complementos alimenticios. Barcelona. 206.

6. Del Arco, J. 2016. Aspectos legales y recomendaciones para la selección (I): características generales y complementos alimenticios. El farmacéutico. 535:29-34.

7. Reglamento (UE) $\mathrm{N}^{\circ} 432 / 2012$ de la Comisión, de 16 de mayo de 2012, por el que se establece una lista de declaraciones autorizadas de propiedades saludables de los alimentos distintas de las relativas a la reducción del riesgo de enfermedad y al desarrollo y la salud de los niños. Diario Oficial de la Unión Europea, núm. 136, de 25 de mayo de 2012, pág. 1-40.

8. Real Decreto 130/2018, de 16 de marzo, por el que se modifica el Real Decreto 1487/2009, de 26 de septiembre, relativo a los complementos alimenticios. Diario Oficial de la Unión Europea, núm. 75, de 27 de marzo de 2018, pág. 33335-33342.

9. Reglamento (CE) No 1170/2009 de la Comisión, de 30 de noviembre de 2009, por la que se modifican la Directiva 2002/46/CE del Parlamento Europeo y del Consejo y el Reglamento (CE) $\mathrm{N}^{\circ}$ 1925/2006 del Parlamento Europeo y del Consejo en lo relativo a las listas de vitaminas y minerales y sus formas que pueden añadirse a los alimentos, incluidos los complementos alimenticios. Diario Oficial de la Unión Europea, núm. 314, de 1 de diciembre de 2009, pág. 36-42. 
10. Complementos alimenticios. En Agencia Española de Consumo, Seguridad Alimentaria y Nutrición. http://www.aecosan.msssi.gob.es/AECOSAN/web/para_el_consumidor/ampliacion/complementos _alimenticios.htm

11. Real Decreto 1808/1991, de 13 de diciembre, por el que se regulan las menciones o marcas que permiten identificar el lote al que pertenece un producto alimenticio. Diario Oficial de la Unión Europea, núm. 308, de 25 de diciembre de 1991, pág. 41511-41511.

12. Reglamento (UE) No 1169/2011 del Parlamento Europeo y del Consejo, de 25 de octubre de 2011, sobre la información facilitada al consumidor. Diario Oficial de la Unión Europea, núm. 304, de 22 de noviembre de 2011, pág. 18-63.

13. Complementos alimenticios: siempre bajo control. En Comunidad de Madrid. http://www.comunidad.madrid/servicios/salud/complementos-alimenticios-siempre-control

14. Reglamento (CE) No 1924/2006 del Parlamento Europeo y del Consejo, de 20 de diciembre de 2006, relativo a las declaraciones nutricionales y de propiedades saludables en los alimentos. Diario Oficial de la Unión Europea, núm. 404, de 30 de diciembre de 2006, pág. 9-25.

15. Reglamento (CE) N 2073/2005 de la Comisión, de 15 de noviembre de 2005, relativo a los criterios microbiológicos aplicables a los productos alimenticios. Diario Oficial de la Unión Europea, núm. 338 , de 22 de diciembre de 2005, pág. 1-26.

16. Metales pesados. 2015 (2 octubre). En Agencia Española de Consumo, Seguridad Alimentaria y Nutrición.

http://www.aecosan.msssi.gob.es/AECOSAN/web/seguridad_alimentaria/subdetalle/metales_pesa dos.htm

17. Reglamento (CE) No $629 / 2008$ de la Comisión, de 2 de julio de 2008 , que modifica el Reglamento (CE) No 1881/2006 por el que se fija el contenido máximo de determinados contaminantes en los productos alimenticios. Diario Oficial de la Unión Europea, núm. 173, de 3 de julio de 2008, pág. 69.

18. Directiva 2001/18/CE del Parlamento Europeo y del Consejo, de 12 de marzo de 2001, sobre la liberación intencional en el medio ambiente de organismos modificados genéticamente y por la que se deroga la Directiva 90/220/CEE del Consejo. Diario Oficial de la Unión Europea, núm. 106, de 17 de abril de 2001, pág. 1-39.

19. Reglamento (CE) No 1829/2003 del Parlamento Europeo y del Consejo, de 22 de septiembre de 2003, sobre alimentos y piensos modificados genéticamente. Diario Oficial de la Unión Europea, núm. 268, de 18 de octubre de 2003, pág. 1-23.

20. Encefalopatías espongiformes transmisibles (EETs). En Agencia Española de Consumo, Seguridad Alimentaria y Nutrición.

http://www.aecosan.msssi.gob.es/AECOSAN/web/seguridad_alimentaria/detalle/eet.htm

21. Encefalopatía espongiforme bovina (EEB). 2018 (6 agosto). En Organización Mundial de Sanidad Animal. https://www.oie.int/es/sanidad-animal-en-el-mundo/enfermedades-de-losanimales/encefalopatia-espongiforme-bovina/

22. Deepak, G., Kamal, K., Ajay, B., et al. 2014. Soft gelatin capsules: development, applications and recent patents. International research journal of inventions in pharmaceutical sciences. 2(3):163:173.

23. International Alliance of Dietary/Food Supplement Associations. 2014. Recomendaciones sobre la vida útil de los complementos alimenticios. Directrices para fabricantes. Bruselas. 41.

24. Organización Mundial de la Salud. 1996. Anexo 5: Directrices para las pruebas de estabilidad de productos farmacéuticos que contienen sustancias medicamentosas bien establecidas en formas farmacéuticas corrientes. Comité de expertos de la OMS en especificaciones para las preparaciones farmacéuticas. 71-89.

25. Legislación Española. 2013 (9 marzo). En Complementos Alimenticios. https://www.complementosalimenticios.info/index.php/conociendo-los-complementosalimenticios/legislacion-espanola

26. Procedimientos. En Agencia Española de Consumo, Seguridad Alimentaria y Nutrición. http://www.aecosan.msssi.gob.es/AECOSAN/web/seguridad_alimentaria/subseccion/procedimient os_registro.htm\#2 